\title{
Endovascular Treatment for Aneurysmal Subarachnoid Hemorrhage with Neurogenic Pulmonary Edema in the Acute Stage
}

\author{
Toshinari MEGURO, Tomoyuki TANABE, Kenichiro MURAOKA, Kinya TERADA, Nobuyuki HIROTSUNE, \\ Shigeki NISHINO
}

Hiroshima City Hospital, Department of Neurological Surgery, Hiroshima, Japan

\section{ABSTRACT}

AIM: Severe neurogenic pulmonary edema (NPE) can occur in a variety of brain insults, including subarachnoid hemorrhage (SAH), and severe case of NPE can cause devastating consequences. But the literature on the treatment strategy about aneurysmal SAH with NPE is very scant. We present that SAH patients with severe NPE, who were treated first by embolization of aneurysm followed by insertion of lumbar spinal drainage, had comparatively good outcome.

MATERIAL and METHODS: We present 12 consecutive cases of aneurysmal SAH with NPE in the acute stage, which were treated by endovascular treatment between April 2002 and December 2012. We classified the patients according to the Hunt and Hess grading system as follows: grade-3 (1 patient), grade-4 (4 patients), and grade-5 (7 patients). All patients needed respiratory management, with the assistance of a ventilator, and underwent endovascular treatment for the ruptured aneurysms within 72 hours from onset. For all the patients, immediately after the endovascular treatment, we performed lumbar spinal drainage.

RESULTS: The pulmonary edema disappeared rapidly after respiratory management and endovascular treatment. The outcomes were as follows: good recovery (GR; 3 patients), moderate disability (MD; 4 patients), severe disability (SD; 3 patients), and death (D; 2 patients). Five patients (42\%) developed pneumonia, and we postponed extubation until recovery from pneumonia. The cause for severe disability and death was symptomatic vasospasm and primary brain damage. No patients had rebleeding from ruptured aneurysms.

CONCLUSION: Endovascular treatment for ruptured aneurysm and placement of lumbar spinal drainage is an excellent treatment option for severe SAH with NPE.

KEYWORDS: Neurogenic pulmonary edema, Endovascular treatment, Aneurysm, Subarachnoid hemorrhage

\section{INTRODUCTION}

$\mathrm{N}$ eurogenic pulmonary edema (NPE) may develop in patients with acute intracranial insult $(3,9)$. Weir reported an incidence of $31 \%$ of clinically apparent pulmonary edema in patients with fatal subarachnoid hemorrhage (SAH), with autopsy confirming pulmonary edema in $78 \%$ of those patients (13). Other reports suggest that NPE manifests in approximately $25 \%$ of patients with SAH $(2,8)$ and is associated with worsened clinical outcome $(6,11)$. But the literature on the treatment strategy about SAH with NPE is very scant.

Endovascular therapy for ruptured intracranial aneurysms has become an important and well-known alternative to surgical clipping, and is relatively a less invasive treatment (4). We previously reported that $4 \mathrm{SAH}$ patients with severe NPE, who were treated first by embolization of aneurysm followed 
by insertion of lumbar spinal drainage, had comparatively good outcome (10). We now have experience in performing this procedure on 12 consecutive cases thus far, including the $4 \mathrm{SAH}$ cases mentioned. To identify the usefulness of this treatment, in this present study, we retrospectively investigated the clinical characteristics and outcome in those 12 patients with SAH and NPE.

\section{MATERIAL and METHODS}

Four hundred and sixty-six patients with aneurysmal SAH underwent treatment in our hospital between April 2002 and December 2012. Computed tomography (CT) scan was used to diagnose SAH. Of the 466 patients, 13 (2.8\%) showed severe NPE. Severe NPE include the presence of all of the following criteria: (i) immediate onset of respiratory failure after ictus; (ii) presence of crackles, which suggested fluid in the lungs by chest auscultation, and frothy pink tracheal fluid; (iii) requirement of endotracheal intubation and respiratory management with a mechanical ventilator; (iv) chest X-ray film on admission revealing interstitial edema in bilateral lung fields; and (v) no previous history of cardiac and lung disease.

After the diagnosis of SAH with severe NPE, all patients underwent endovascular treatment for ruptured aneurysms under general anesthesia, following diagnostic angiography, as soon as the respiratory condition became stable, as judged by the anesthesiologist. After the endovascular treatment, we placed continuous lumbar spinal drainage for early discharge of SAH and control of intracranial pressure (ICP).

\section{RESULTS}

Of the 13 patients who presented with SAH and severe NPE, 12 were treated by endovascular treatment within 3 days of ictus, and 1 patient, with intracerebral hematoma, was treated by surgical clipping and removal of hematoma. Figure 1 shows the initial brain CT scan of the 12 patients. All patients had diffuse SAH and 3 of 12 patents showed enlargement of lateral ventricles, but every patient did not have so much volume of $\mathrm{SAH}$ and they had no intracerebral hematoma (Figure 1). Four of 12 patients (cases 2, 3, 6, and 7) were previously reported (10). Mean age of the 12 patients was 52 (range, 31-74) years, and 6 patients $(50 \%)$ were male. The Hunt and Hess grades on admission were as follows: grade-3 (1 patient), grade-4 (4 patients), and grade-5 (7 patients). The locations of aneurysm in the 12 patients (in parentheses) were as follows: basilar top (2), posterior communicating artery (1), middle cerebral artery (1), anterior cerebral artery (1), anterior communicating artery (2), basilar-anterior inferior cerebellar artery (1), internal carotid-posterior communicating (2), and vertebral artery dissecting aneurysm (2). We treated the 2 patients with vertebral artery dissecting aneurysm by internal trapping of vertebral artery, and the remaining 10 patients by aneurysmal sac embolization. All patients required early intratracheal

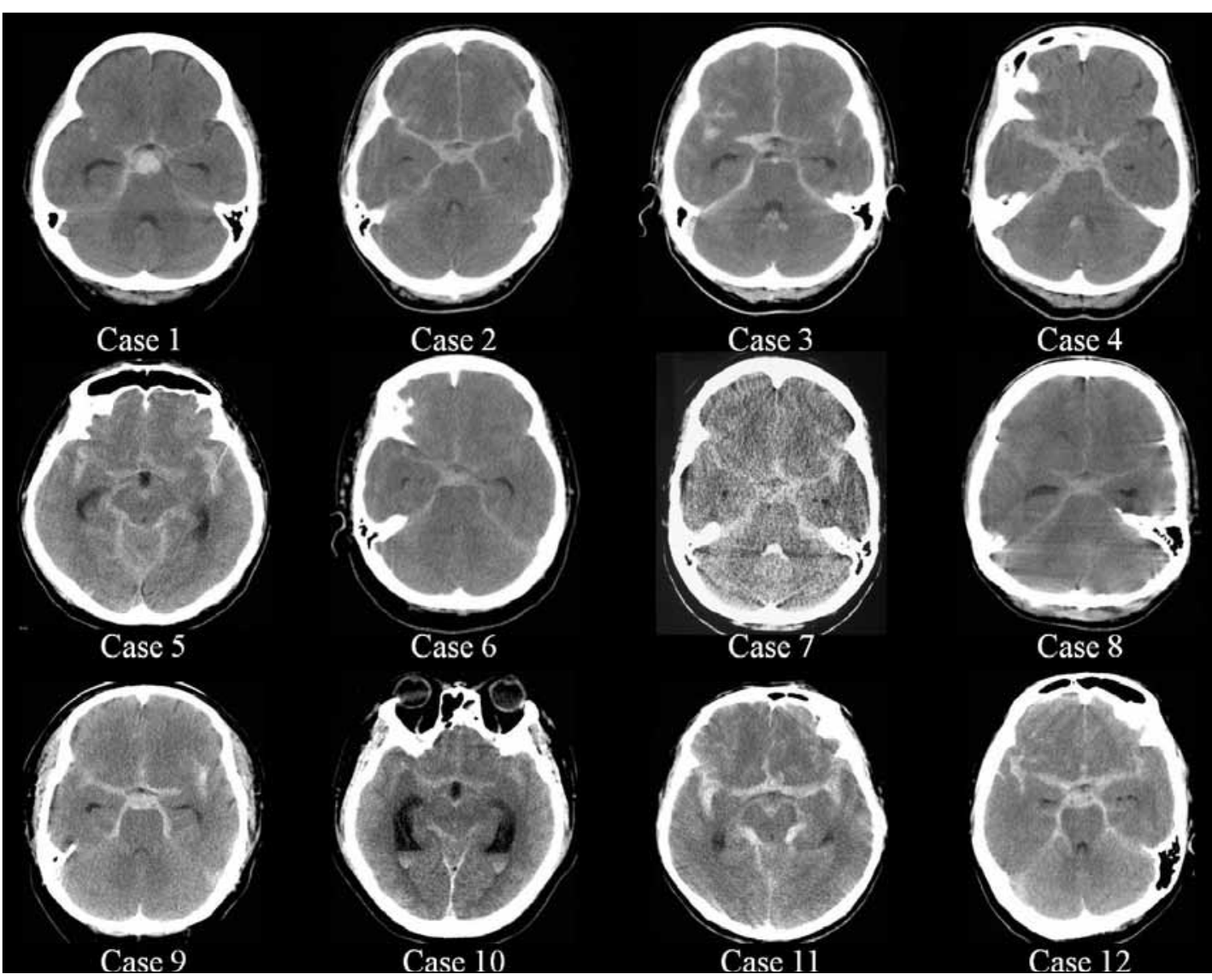

Figure 1: Initial head CT scans of 12 patients shows diffuse subarachnoid hemorrhage. 
intubation and successfully underwent endovascular treatment following diagnostic angiography within $72 \mathrm{~h}$ from onset of ictus. Immediately after the endovascular treatment, we placed continuous lumbar spinal drainage for early discharge of SAH and control of ICP. All patients required respiratory management with a mechanical ventilator. Pulmonary edema

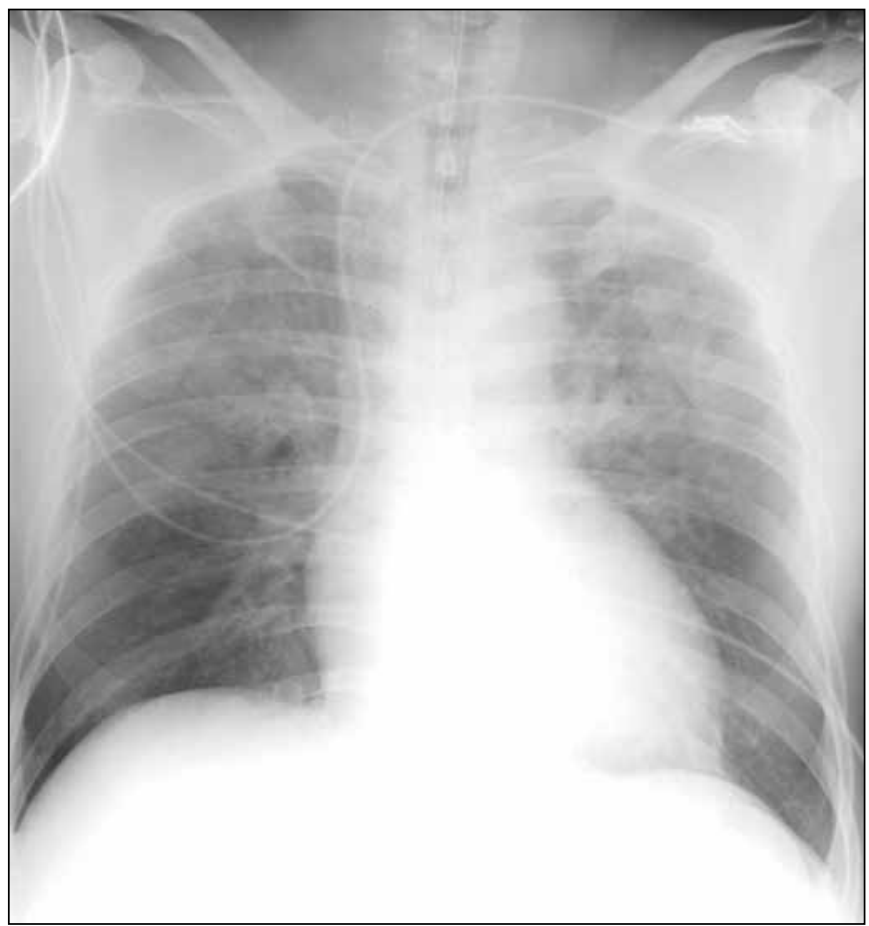

Figure 2: Chest $X$-ray reveals diffuse pulmonary infiltrates in the lung indicating the presence of pulmonary edema. disappeared in all patients within 6 days, but 5 patients (42\%) developed complicated pneumonia. We postponed extubation until recovery from pneumonia, and 1 patient had tracheotomy for pneumonia and prolonged disturbance in consciousness. We maintained the continuous lumbar spinal drainage for 7 to 14 days (mean 10 days). Three of the patients achieved good recovery, 4 patients suffered mild disability, 3 patients suffered severe disability due to the symptomatic vasospasm, and 2 patients died of primary brain damage after 6 and 9 days, respectively, from onset. Mean follow-up periods were 38 months, and no aneurysms reruptured in this period.

\section{- ILLUSTRATIVE CASE}

\section{Case 9}

A 51-year-old man was admitted to our hospital with a sudden onset of unconsciousness. He presented with coma and respiratory dysfunction with pinkish foamy sputum on admission. CT scan revealed diffuse subarachnoid hemorrhage, and a chest roentgenogram revealed diffuse pulmonary infiltrates in the bilateral lung field indicating the presence of pulmonary edema (Figure 2). He was also intubated immediately after admission and was controlled by continuous positive-pressure ventilation with positive end-expiratory pressure. Angiography was performed under general anesthesia after $4 \mathrm{~h}$ of onset. Left vertebral angiogram revealed a saccular aneurysm at the top of the basilar artery (Figure 3A). The patient underwent endovascular embolization with detachable coils following diagnostic angiography. Complete occlusion of the aneurysm was performed (Figure 3B). Immediately after the embolization, continuous lumbar spinal drainage was inserted and placed for 14 days.
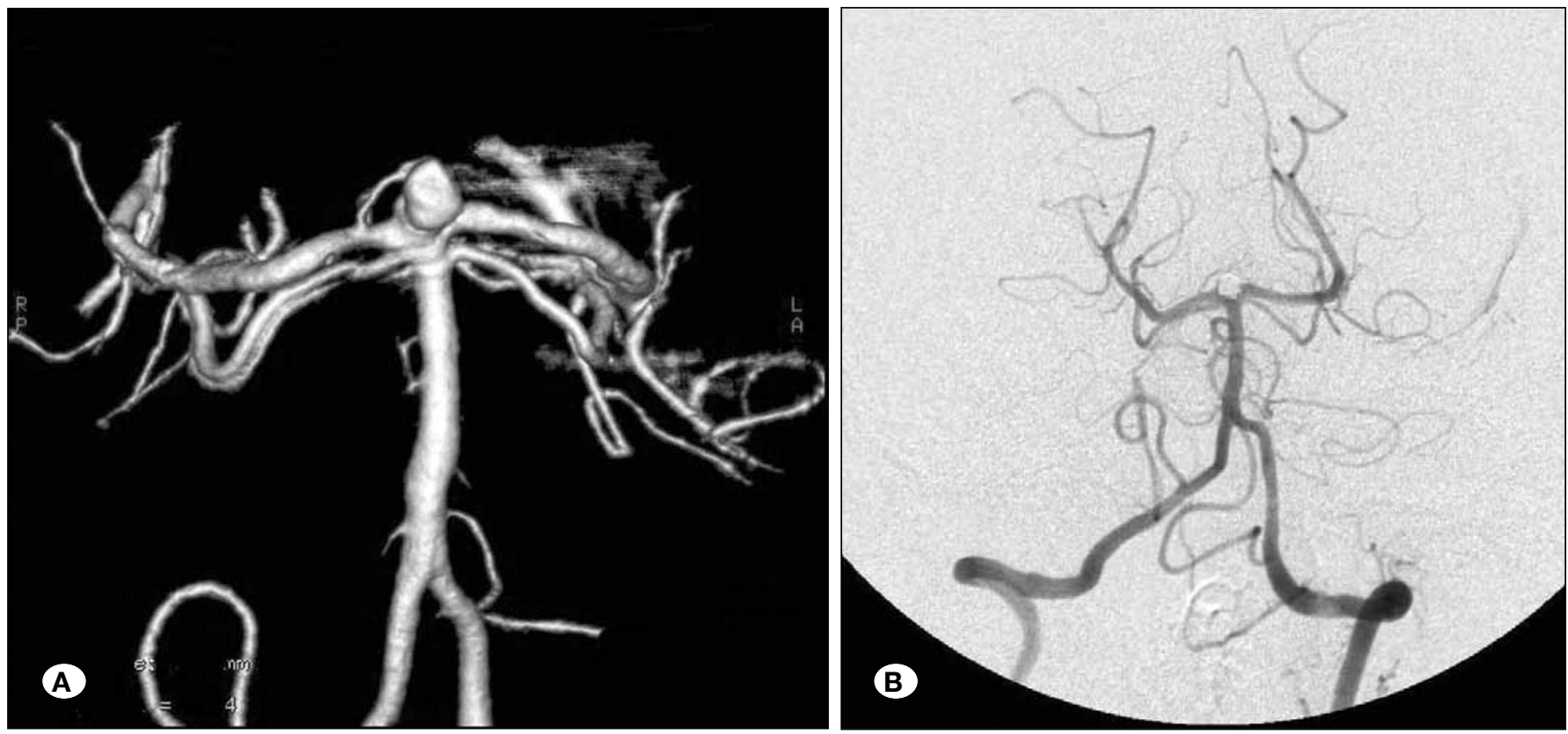

Figure 3: A) 3D-digital subtraction angiography of the right vertebral injection reveals top of basilar artery aneurysm. B) The aneurysm was completely embolized by detachable coils. 


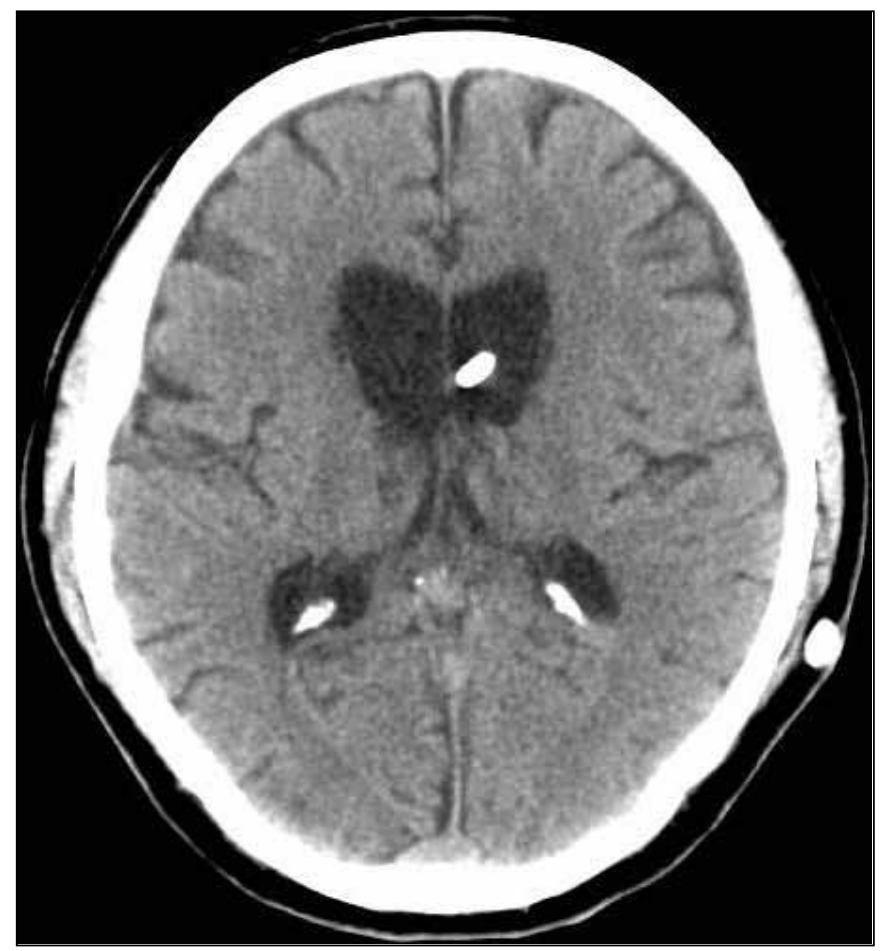

Figure 4: CT scan after ventriculoperitoneal shunt shows mild brain atrophy.

The pulmonary edema findings disappeared rapidly after endovascular treatment, but aspiration pneumonia developed as a complication. Therefore, extubation was done after 8 days from onset. Serial chest roentgenograms revealed complete resolution on the tenth day. After removal of the spinal drainage, hydrocephalus developed. Ventriculoperitoneal shunt was performed on day 24 , but neurological recovery was incomplete, and recent memory disturbance was present due to primary brain damage. CT scan on the $40^{\text {th }}$ day from onset revealed mild brain atrophy (Figure 4).

\section{DISCUSSION}

NPE is usually defined as an acute pulmonary edema occurring shortly after neurogenic insult $(3,9)$. Frequent causes of NPE are traumatic head injury or aneurysmal SAH. Several mechanisms have been implicated in the pathogenesis of NPE. Sudden increase in ICP induces massive sympathetic discharge (catecholamine surge) that causes systemic arterial hypertension and peripheral and pulmonary microvascular vasoconstriction. Severe generalized vasoconstriction leads to a shift of intravascular volume from high-resistance systemic circulation to low-resistance pulmonary circulation. The resultant hydrostatic force along with an already increased capillary permeability causes formation of pulmonary edema $(5,7,12)$. However, the exact interactions remain unknown.

Muroi, et al. reported that patients with NPE presented significantly more often with clinically severe hemorrhage compared with patients without NPE (11). Nevertheless, there have been few reports regarding the best management for aneurysmal SAH with NPE in the acute stage. There is a therapeutic dilemma between the policy of early aneurysm surgery and the successful management of pulmonary edema during radical intervention. Yabumoto et al reported a case of aneurysmal SAH with NPE (14). They insisted on early and aggressive management of the ruptured aneurysm and NPE, and proposed that the syndrome of NPE should not be an obstacle to radical intervention when cardiorespiratory control can maintain the minimal anesthetic limit. In the present study, the authors also carried out an early and aggressive management of the ruptured aneurysms. Although 11 of 12 patients $(92 \%)$ were poor grade SAH (Hunt and Hess grade IV or V), 7 of 12 patients (58\%) were good recovery or mild disability in Glasgow Outcome Scale at discharge. This is quite a good outcome. The initial head CT scan showed that every patient did not have so much volume of SAH, and NPE might worsen the SAH grading. Even a little volume of SAH might cause severe NPE.

Although the conventional treatment of pulmonary edema are tracheal intubation, controlled ventilation with supplemental oxygen, moderate positive end-expiratory pressure, diuretics, etc., the essential management of NPE is based on the rapid control of the triggering central neurological insult, and therefore, the primary goal therapeutic approach needs to be focused on decreasing ICP (1). Recently, endovascular treatment has been recognized as a less invasive treatment for cerebral aneurysm when compared to direct surgical clipping (1). The most important advantages of endovascular treatment for aneurysmal SAH are that it can immediately follow the diagnostic angiography and that the period required for intervention is shorter than that for direct clipping (4). Lumbar spinal drainage also can be performed in a shorter time than ventricular drainage. Rapidly control of ICP after endovascular treatment could contribute to recovery from NPE. We therefore suggest that early endovascular intervention for ruptured aneurysm followed by lumbar drainage of cerebrospinal fluid for decreasing ICP should be the first choice for poor-grade aneurysmal SAH with NPE.

In the acute stage, unconscious patients are at risk of aspiration of gastric contents, or using of ventilation for treatment of NPE might induce nosocomial infection. In either event, the patients of aneurysmal SAH with NPE might develop pneumonia. Of 12 patients, 5 (42\%) developed pneumonia after improvement of NPE, and we postponed extubation until recovery from pneumonia. Fortunately, all 5 patients were successfully treated with antibiotics. However, pneumonia is a severe complication that must not be ignored.

\section{CONCLUSION}

Early endovascular treatment of ruptured aneurysm and placement of lumbar spinal drainage is an excellent treatment option for severe SAH with NPE.

\section{REFERENCES}

1. Ahrens J, Capelle HH, Przemeck M: Neurogenic pulmonary edema in a fatal case of subarachnoid hemorrhage. J Clin Anesth 20: 129-132, 2008 
2. Baumann A, Audibert G, McDonnell J, Mertes PM: Neurogenic pulmonary edema. Acta Anaesthesiol Scand 51:447-455, 2007

3. Carlson RW, Schaeffer RC Jr, Michaels SG, Weil MH: Pulmonary edema following intracranial hemorrhage. Chest 75: 731-734, 1979

4. Ezura M, Takahashi A, Ogasawara K, Yoshimoto T: Intraaneurysmal GDC embolization followed by intrathecal tPA administration for poor-grade basilar tip aneurysm. Surg Neurol 47: 144-147; discussion 147-148, 1997

5. Hoff JT, Nishimura M, Garcia-Uria J, Miranda S: Experimental neurogenic pulmonary edema. Part 1: The role of systemic hypertension. J Neurosurg 54: 627-631, 1981

6. Kahn JM, Caldwell EC, Deem S, Newell DW, Heckbert SR, Rubenfeld GD: Acute lung injury in patients with subarachnoid hemorrhage: Incidence, risk factors, and outcome. Crit Care Med 34: 196-202, 2006

7. Komiyama M, Tamura K, Nagata $Y, F u ~ Y$, Yagura H, Yasui T: Aneurysmal rupture during angiography. Neurosurgery 33: 798-803, 1993
8. Macmillan CS, Grant IS, Andrews PJ: Pulmonary and cardiac sequelae of subarachnoid haemorrhage: Time for active management? Intensive Care Med 28: 1012-1023, 2002

9. McLaughlin N, Bojanowski MW, Girard F, Denault A: Pulmonary edema and cardiac dysfunction following subarachnoid hemorrhage. Can J Neurol Sci 32: 178-185, 2005

10. Meguro T, Terada K, Hirotsune N, Nishino S, Asano T, Manabe $\mathrm{T}$ : Early embolization for ruptured aneurysm in acute stage of subarachnoid hemorrhage with neurogenic pulmonary edema. Interv Neuroradiol 13 Suppl 1: 170-173, 2007

11. Muroi C, Keller M, Pangalu A, Fortunati M, Yonekawa Y, Keller E: Neurogenic pulmonary edema in patients with subarachnoid hemorrhage. J Neurosurg Anesthesiol 20: 188-192, 2008

12. Nathan MA, Reis DJ: Fulminating arterial hypertension with pulmonary edema from release of adrenomedullary catecholamines after lesions of the anterior hypothalamus in the rat. Circ Res 37: 226-235, 1975

13. Weir BK: Pulmonary edema following fatal aneurysm rupture. J Neurosurg 49: 502-507, 1978

14. Yabumoto M, Kuriyama T, Iwamoto M, Kinoshita T: Neurogenic pulmonary edema associated with ruptured intracranial aneurysm: Case report. Neurosurgery 19: 300-304, 1986 